\title{
Ready Queue Mean Time Estimation in Lottery Scheduling using Auxiliary Variables in Multiprocessor Environment
}

\author{
D. Shukla \\ Deptt. of Mathematics and Statistics \\ Dr.H.S.Gour Central University \\ Sagar, (M.P), INDIA
}

\author{
Anjali Jain \\ Deptt. of Computer Science and Applications \\ Dr.H.S.Gour Central University \\ Sagar ,(M.P.), INDIA
}

\begin{abstract}
The ready queue estimation problem appears when many processes remain in the ready queue after the sudden failure. The system manager has to decide immediately how much further time is required to process all the remaining jobs in the ready queue. In lottery scheduling, this prediction is possible with the help of sampling techniques. To strengthen the prediction methodology, the auxiliary source of data is often utilized. This paper considers the three additional data sources as (i) process size (ii) process priority and (iii) process expected time. The Ratio method, existing in sampling literature, is used to predict the time required for remaining jobs after failure. A comparative study between different auxiliary sources has been made. It is found that highly correlated source of auxiliary information provides better processing time prediction.
\end{abstract}

Keywords: Scheduling, Ratio Estimator, Bias, Variance, Confidence Interval, Ready Queue, Expected Time (et), Size(s), Priority (p).

\section{INTRODUCTION}

Suppose there are $\mathrm{n}$ processors in multiprocessor and multi-user environment and a large number of processes, say $\mathrm{N}(\mathrm{N}>\mathrm{n})$, are in the ready queue. Assume the scheduler adopts lottery scheduling to choose randomly any $n$ processes(jobs) from the ready queue and allocates to $\mathrm{n}$ processors in the sequential manner. In lottery scheduling, each process is pre-allocated with one (or more) lottery tickets which determine the possibility of that process priority to use the CPU. At each schedule point, a lottery ticket- number is drawn and process in the ready queue with the winning ticket gets the opportunity of CPU utilization. Every job has equal chance of being represented for processing if it has only one pre-allocated ticket. Special feature of Lottery scheduling is that it does not suffer from starvation.

The problem is when $\mathrm{N}$ large, the congestion occurs and many processes have to wait until called by lottery manner. During processing through lottery scheduling, if the system fails suddenly due to unavoidable reasons, the system manager has to arrange backup provisions( or recovery) immediately . His problem, at this juncture, is to know how much probable time is required to finish up the remaining processes in queue? This prediction is an uncertain evaluation and needs theoretical probability plus sampling mechanism both to resolve the issue. This paper takes into account same problem and presents a method for predicting the processing time interval.

Shukla and Jain [16] picked up multiprocessor environment with usual lottery scheduling and discussed a procedure to obtain ready queue remaining time estimate. Shukla et al. [14] suggested systematic lottery scheduling scheme to improve upon the prediction of ready queue processing time. Shukla et al. [15] recommended similar when processes are grouped according to specific criteria in different queues and obtained well-predicted confidence intervals. Shukla et al. [17] introduced process-size based priority scheme for the ready queue remaining time length prediction and proved better than usual lottery scheduling prediction.

This paper suggests incorporating the auxiliary sources of information related to waiting processes in the ready queue like process size, response time, pre-set priority etc. for the efficient time interval prediction

\section{A REVIEW}

Cochran [3] contains basics of sampling methods with applications on multiple data. David [7] extended lottery scheduling by a proportional share resource management algorithm to provide the better performance assurance. $\mathrm{He}$ introduced dynamic tickets in lottery scheduler to improve the interactive response time and to reduce kernel lock contention. Raz et al. [9] presented a procedure of deciding priorities among jobs maintaining fairness in the selection procedure. Shukla and Jain [4], [12] suggested for the Markov chain based study for the behavior of scheduler in the multilevel queue scheduling. Shukla and Jain [5],[6] described procedures of analysis for the Thread scheduling and the Deficit Round Robin Alternated (DRRA) scheduling using the Markov Chain Model. Shukla and Ojha [13] performed an analysis for the multilevel queue scheduling with the special effect of data model. Waldspurger [1],[2] proposed lottery scheduling ticket/currency framework that can be accommodated with scheduling mechanism. He discussed the proportional share resource management technique useful for lottery scheduling. Yiping [22],[23] develop a queuing theory model to predict system behavior and CPU queue length in Microsoft NT , Windows 2000 and devised fair share scheduling which guarantees the application performance by allocating the share of system resources among competing workloads. Some other useful similar contributions are [8], [10], [11], [18], [19], [20], [21].

\section{MOTIVATION}

It is common believe that more input information provide better prediction subject to condition if information are relevant and related. If a large number of processes are in ready queue then to predict for possible CPU utilization time, additional sources of correlated information may reduce the length of computed time interval. Using motivation with this thought and using estimation technique of sampling theory with auxiliary information, this paper suggests a new useful approach and compares with Shukla, Jain and Chowhary [16]. 


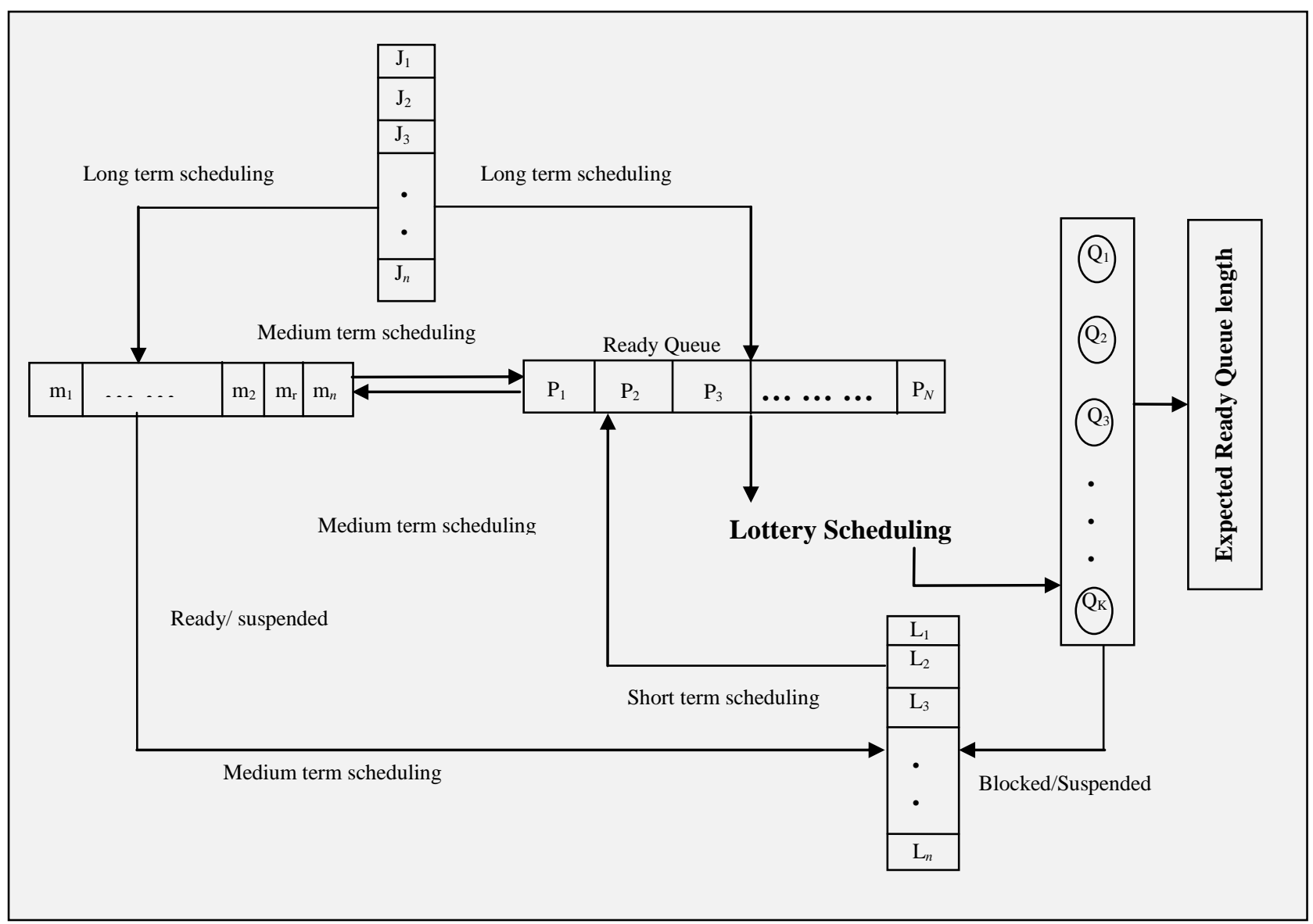

\section{PROCESSOR STRUCTURE AND NOTATIONS}

Let $Q_{1}, Q_{2}, Q_{3}$ .. $Q_{k}$ be k processors who take intake from the ready queue containing $P_{1}, P_{2}, P_{3} \ldots \ldots \ldots P_{N}$ processes $(\mathrm{n}<\mathrm{N})$. Processes are related to long, medium and short term scheduling queues all transferring to ready queue. When a process remains blocked/suspended, they are back to respective queue. The Figure 1 shows the diagram of scheduling process structure with $\mathrm{k}$ processors.

Let $Y_{i}$ be the CPU burst time of each process and $X_{1 i}, X_{2 i}, X_{3 i}$ are three auxiliary variables like size of process, process priority and processes expected time( time interval between arrival time and processor entering time also called response time) respectively. The mean entire ready queue CPU burst time(Y) of $N$ processes along with auxiliary characters $\left(\mathrm{X}_{1}, \mathrm{X}_{2}, \mathrm{X}_{3}\right)$ in the ready queue is:

$$
\begin{aligned}
& \bar{Y}=\frac{1}{N} \sum_{i=1}^{N} Y_{i} \\
& \bar{X}_{1}=\frac{1}{N} \sum_{i=1}^{N} X_{1 i} \\
& \bar{X}_{2}=\frac{1}{N} \sum_{i=1}^{N} X_{2 i}
\end{aligned}
$$

$$
\bar{X}_{3}=\frac{1}{N} \sum_{i=1}^{N} X_{3 i}
$$

\subsection{MODIFIED MULTIPROCESSOR LOTTERY SCHEDULING [As Shukla, Jain and Choduary [16])}

Step i

Step ii

Step iii

Step iv

Step v
When a process enters into ready queue, it is allotted a random number (in specified range).

$$
\text { Each processor } Q_{1}, Q_{2}, Q_{3} \ldots \ldots \ldots . . Q_{K}
$$

generates unique and uncommon random number in similar specified range stated in Step I.

Matching of both random numbers takes place between process and processor. If both random numbers are same for a process in ready queue, it is assigned that processor.

Processor either blocks or processes the job. It selects another process in random manner.

After when one job processed completely or partially processors generate time consumed by it in processing

$$
\begin{aligned}
& \text { as } y_{i} \text { (time by } j^{\text {th }} \text { processor) where } \\
& (j=1,2,3 \ldots \ldots k) .
\end{aligned}
$$


Step vi Corresponding to time consumed by $\mathrm{n}$ processes as $y_{1}, y_{2}, y_{3} \ldots \ldots \ldots y_{n}$ there are auxiliary variables

$$
\begin{aligned}
& X_{1}, X_{2}, X_{3} \text { in the form }\left(x_{11}, x_{12}, x_{13 \ldots \ldots . . . .} x_{1 n}\right) \text {; } \\
& \left(x_{21}, x_{22}, x_{23} \ldots . x_{2 n}\right) ;\left(x_{31}, x_{32}, x_{33} \ldots \ldots x_{3 n}\right) \\
& \text { respectively. }
\end{aligned}
$$

\section{ESTIMATION OF READY QUEUE PROCESSING TIME (as per [16])}

Suppose $P_{1}, P_{2}, P_{3} \ldots \ldots P_{N}$ processes present in the ready queue of size $N$ and until breakdown occurs, $n$ processes $(\mathrm{n}<\mathrm{N})$ have already processed in random manner through lottery scheduling by $\mathrm{k}$ processors. Each Processor generates timeconsumed by $\mathrm{i}^{\text {th }}$ during processing as $y_{i}$. The corresponding auxiliary variable source value is $\mathrm{x}_{\mathrm{i}}$. Now, we have some basic results discussed below used for prediction purpose. The ratios estimates of the population total $Y$, the population mean, $\bar{Y}$ and the population ratio $\frac{Y}{X}$ are, respectively $\hat{Y}_{R}=\frac{\bar{y}}{\bar{x}} X$, $\hat{\bar{Y}}_{R}=\frac{\bar{y}}{\bar{x}} \bar{X}, \hat{R}=\frac{\bar{y}}{\bar{x}}$.

In a simple random sample of size $\mathrm{n}$ ( $\mathrm{n}$ large) variances of these estimates are

$V\left(\hat{Y}_{R}\right)=\frac{N^{2}(1-f)}{n}\left[\frac{\sum_{i=1}^{N}\left(y_{i}-R x_{i}\right)^{2}}{N-1}\right]$

$V\left(\hat{\bar{Y}}_{R}\right)=\frac{1-f}{n}\left[\frac{\sum_{i=1}^{N}\left(y_{i}-R x_{i}\right)^{2}}{N-1}\right]$

$V(\hat{R})=\frac{1-f}{n \bar{X}^{2}}\left[\frac{\sum_{i=1}^{N}\left(y_{i}-R x_{i}\right)^{2}}{N-1}\right]$

Where $\mathrm{f}=\mathrm{n} / \mathrm{N}$ is the sampling fraction. So for a sample of processes variance is

$$
v\left(\hat{Y}_{R}\right)=\frac{1-n / N}{n \bar{x}^{2}}\left[\frac{\sum_{i=1}^{n}\left(y_{i}-R x_{i}\right)^{2}}{n-1}\right]
$$

$v\left(\hat{Y}_{R}\right)=\frac{N-n}{n N} \frac{1}{\bar{x}^{2}} \frac{\sum_{i=1}^{n}\left(y_{i}-R x_{i}\right)^{2}}{n-1}$

$\hat{\bar{Y}}_{R}=\bar{X} \hat{R}, \hat{Y}_{R}=N \bar{X} \hat{R}$

We get

$$
\begin{aligned}
& V\left(\hat{Y}_{R}\right)=\frac{N^{2}(1-f)}{n(N-1)} \sum_{i=1}^{N}\left[\left(y_{i}-\bar{Y}\right)-R\left(x_{i}-\bar{X}\right)\right]^{2} \\
& =\frac{N^{2}(1-f)}{n(N-1)}\left[\sum\left(y_{i}-\bar{Y}\right)^{2}+R^{2} \sum\left(x_{i}-\bar{X}\right)^{2}-2 R \sum\left(y_{i}-\bar{Y}\right)\left(x_{i}-\bar{X}\right)\right]
\end{aligned}
$$

This leads to result (using Appendix $-\mathrm{A}$ )

$$
V\left(\hat{Y}_{R}\right)=\frac{N^{2}(1-f)}{n}\left(S_{y}{ }^{2}+R^{2} S_{x}{ }^{2}-2 R \rho S_{y} S_{x}\right)
$$

An equivalent form is

$V\left(\hat{Y}_{R}\right)=(1-f) \frac{Y^{2}}{n}\left(\frac{S_{y}^{2}}{\bar{Y}^{2}}+\frac{S_{x}^{2}}{\bar{X}^{2}}-\frac{2 S_{y} S_{x}}{\overline{Y X}}\right)$

Where is $S_{y x}=\rho S_{y} S_{x}$ the covariance between $\mathrm{y}_{\mathrm{i}}$ and $\mathrm{x}_{\mathrm{i}}$. This relation may also be written as

$$
V\left(\hat{Y}_{R}\right)=(1-f) \frac{Y^{2}}{n}\left(C_{y y}+C_{x x}-2 C_{y x}\right)
$$

Where $\mathrm{C}_{\mathrm{yy}}, \mathrm{C}_{\mathrm{xx}}$ are the square of the coefficients of variation

(cv) of $\mathrm{y}_{\mathrm{i}}$ and $\mathrm{x}_{\mathrm{i}}$ respectively and $\mathrm{c}_{\mathrm{xy}}$ is the relative covariance. Since $\hat{Y}_{R} \hat{\bar{Y}}_{R}$ and $\hat{R}$ differ only by known multipliers, the coefficient of variation (i.e., the standard error divided by the quantity being estimated) is the same for all three estimates. From the square of this $(c v)$ is

$(c v)^{2}=\frac{V\left(\hat{Y}_{R}\right)}{Y^{2}}=\frac{1-f}{n}\left(C_{Y Y}+C_{x x}-2 C_{y x}\right)$

The quantity $(c v)^{2}$ has been has been called the relative variance. Its use avoids repetition of variance formulas for related quantities like the estimated population total and mean.

\subsection{ESTIMATION OF THE VARIANCE FROM A SAMPLE}

From equation (1) we get estimation of the variance from a sample as a sample estimate of variance.

For the estimated variance, this gives 
$v\left(\hat{Y}_{R}\right)=\frac{N^{2}(1-f)}{n(n-1)} \sum_{i=1}^{n}\left(y_{i}-\hat{R} x_{i}\right)^{2}$

$v\left(\hat{Y}_{R}\right)=\frac{N^{2}(1-f)}{n(n-1)}\left(\sum y_{i}^{2}+\hat{R}^{2} \sum x_{i}^{2}-2 \sum y_{i} x_{i}\right)$

$=\frac{N^{2}(1-f)}{n}\left(s_{y}^{2}+\hat{R}^{2} s_{x}^{2}-2 \hat{R} s_{y x}\right)$

$s_{y x}=\sum\left(y_{i}-\bar{y}\right)\left(x_{i}-\bar{x}\right) /(n-1)$

is sample covariance between $\mathrm{y}_{\mathrm{i}}$ and $\mathrm{x}_{\mathrm{i}}$.
The Confidence Interval are:

$$
\begin{aligned}
& Y: \hat{Y}_{R} \pm z \sqrt{v\left(\hat{Y}_{R}\right)} \\
& R: \hat{R} \pm z \sqrt{v(\hat{R})}
\end{aligned}
$$

Where

$$
z=\frac{\bar{y}-R \bar{x}}{\sqrt{[(N-n) / N n] \sqrt{S_{y}^{2}+R^{2} S_{x}^{2}-2 R S_{y x}}}}
$$

\section{NUMERICAL DATA}

Consider 30 processes in ready queue at a time whose size measure $X_{1}$ is given in terms of bytes, priority $X_{2}$ and arrival time $X_{3}$ in second's are given. If we assume that all the processes are processed completely in the ready queue, the CPU

\begin{tabular}{|c|c|c|c|c|}
\hline $\begin{array}{l}\text { Process } \\
\text { ID }\end{array}$ & $\begin{array}{l}\text { Size Parameter } \\
\left(\mathbf{X}_{1}\right)\end{array}$ & $\begin{array}{l}\text { Expected Time(sec) } \\
\qquad\left(\mathbf{X}_{2}\right)\end{array}$ & $\begin{array}{l}\text { Process Priorities } \\
\left(\mathbf{X}_{3}\right)\end{array}$ & $\begin{array}{c}\text { CPU Burst } \\
\text { Time } \\
\left(\mathbf{Y}_{\mathbf{i}}\right)\end{array}$ \\
\hline 1 & 210 & 45 & 2 & 30 \\
\hline 2 & 897 & 07 & 9 & 20 \\
\hline 3 & 312 & 16 & 7 & 112 \\
\hline 4 & 171 & 34 & 2 & 40 \\
\hline 5 & 461 & 56 & 8 & 59 \\
\hline 6 & 290 & 22 & 2 & 60 \\
\hline 7 & 379 & 43 & 1 & 30 \\
\hline 8 & 220 & 33 & 0 & 43 \\
\hline 9 & 470 & 50 & 1 & 101 \\
\hline 10 & 636 & 49 & 4 & 69 \\
\hline 11 & 455 & 55 & 9 & 138 \\
\hline 12 & 682 & 10 & 6 & 43 \\
\hline 13 & 952 & 03 & 3 & 109 \\
\hline 14 & 574 & 29 & 4 & 26 \\
\hline 15 & 536 & 30 & 3 & 74 \\
\hline 16 & 416 & 02 & 3 & 89 \\
\hline 17 & 788 & 5 & 1 & 123 \\
\hline 18 & 902 & 49 & 7 & 67 \\
\hline 19 & 623 & 46 & 8 & 58 \\
\hline 20 & 563 & 26 & 6 & 84 \\
\hline 21 & 111 & 37 & 9 & 143 \\
\hline 22 & 341 & 20 & 3 & 29 \\
\hline 23 & 775 & 51 & 9 & 147 \\
\hline 24 & 913 & 27 & 4 & 94 \\
\hline 25 & 745 & 13 & 9 & 131 \\
\hline 26 & 130 & 19 & 9 & 79 \\
\hline 27 & 877 & 8 & 9 & 46 \\
\hline 28 & 927 & 58 & 3 & 59 \\
\hline 29 & 424 & 31 & 8 & 72 \\
\hline 30 & 356 & 60 & 4 & 22 \\
\hline
\end{tabular}
burst time $\mathrm{Y}$ is mentioned against them.

Table 1: Incoming processes parameters and auxiliary information in ready queue 
Table 2: Processes parameters in ready queue

\begin{tabular}{|c|c|c|c|c|c|c|c|c|c|c|}
\hline Processes & \multicolumn{5}{|c|}{ Sampled Processes I } & \multicolumn{5}{|c|}{ Sampled Processes II } \\
\hline Processes & $\mathrm{P}_{9}$ & $\mathrm{P}_{18}$ & $\mathrm{P}_{30}$ & $\mathrm{P}_{24}$ & $\mathrm{P}_{13}$ & $P_{20}$ & $P_{27}$ & $P_{11}$ & $P_{1}$ & $\mathrm{P}_{22}$ \\
\hline CPU Burst Time $Y_{i}$ & 101 & 67 & 22 & 94 & 109 & 84 & 40 & 138 & 30 & 29 \\
\hline Processes Size $X_{1}$ & 470 & 902 & 356 & 913 & 952 & 563 & 171 & 455 & 210 & 341 \\
\hline Expected Time $\mathrm{X}_{2}$ & 50 & 49 & 60 & 27 & 03 & 26 & 34 & 55 & 45 & 20 \\
\hline Priorities $\mathbf{X}_{3}$ & 01 & 07 & 04 & 04 & 03 & 05 & 02 & 05 & 02 & 02 \\
\hline
\end{tabular}

Table 3: Processes parameters in ready queue

\begin{tabular}{|c|c|c|c|c|c|c|c|c|c|c|}
\hline Processes & \multicolumn{5}{|c|}{ Sampled Processes III } & \multicolumn{5}{|c|}{ Sampled Processes IV } \\
\hline Processes & $\mathrm{P}_{15}$ & $\mathrm{P}_{23}$ & $\mathrm{P}_{5}$ & $\mathrm{P}_{10}$ & $\mathrm{P}_{29}$ & $\mathrm{P}_{30}$ & $\mathrm{P}_{6}$ & $\mathrm{P}_{17}$ & $\mathrm{P}_{25}$ & $\mathrm{P}_{15}$ \\
\hline CPU Burst Time $Y_{i}$ & 74 & 147 & 59 & 69 & 72 & 22 & 60 & 123 & 131 & 74 \\
\hline Processes Size $X_{1}$ & 536 & 775 & 461 & 636 & 424 & 356 & 290 & 788 & 745 & 536 \\
\hline Expected Time $\mathbf{X}_{2}$ & 30 & 51 & 56 & 49 & 31 & 60 & 22 & 05 & 13 & 30 \\
\hline Priorities $X_{3}$ & 03 & 09 & 05 & 04 & 08 & 04 & 02 & 01 & 09 & 03 \\
\hline
\end{tabular}

Table 4: Processes parameters in ready queue

\begin{tabular}{|c|c|c|c|c|c|c|c|c|}
\hline $\begin{array}{l}\text { Sampled } \\
\text { Processes }\end{array}$ & $\begin{array}{l}\text { Sampled Process } \\
\qquad(k=5)\end{array}$ & $\sum X_{s}$ & $\sum X_{e t}$ & $\sum X_{p}$ & $\sum Y_{p t}$ & $\hat{R}_{s}$ & $\hat{R}_{e t}$ & $\hat{R}_{p}$ \\
\hline 1. & $\mathrm{P}_{9}, \mathrm{P}_{18}, \mathrm{P}_{30}, \mathrm{P}_{24}, \mathrm{P}_{13}$ & 3593 & 189 & 19 & 393 & 0.1093 & 2.07 & 20.68 \\
\hline 2. & $P_{20}, P_{27}, P_{11}, P_{1}, \mathrm{P}_{22}$ & 2446 & 72 & 16 & 321 & 0.1844 & 1.78 & 20.06 \\
\hline 3. & $\mathrm{P}_{15}, \mathrm{P}_{23}, \mathrm{P}_{5}, \mathrm{P}_{10}, \mathrm{P}_{29}$ & 2832 & 217 & 29 & 421 & 0.1486 & 1.94 & 14.51 \\
\hline 4. & $\mathrm{P}_{30}, \mathrm{P}_{6}, \mathrm{P}_{17}, \mathrm{P}_{25}, \mathrm{P}_{15}$ & 2715 & 130 & 19 & 410 & 0.1510 & 3.15 & 21.57 \\
\hline
\end{tabular}


Table 5: Confidence Intervals with three auxiliary sources of information

\begin{tabular}{|c|c|c|c|c|}
\hline $\begin{array}{c}\text { Sampled } \\
\text { Processes }\end{array}$ & $\hat{\bar{Y}}_{p t}$ & $\begin{array}{c}\text { Confidence Intervals } \\
\text { In case I } \\
\mathbf{X}_{\mathbf{1}}: \text { Size }\end{array}$ & $\begin{array}{c}\text { Confidence Intervals } \\
\text { In case II } \\
\mathbf{X}_{\mathbf{2}} \text { :Expected Time } \\
(0-226.70)\end{array}$ & $\begin{array}{c}\text { Confidence Intervals } \\
\text { In case III } \\
\mathbf{X}_{\mathbf{3}}: \text { Priority }\end{array}$ \\
\hline 1. & 72.16 & $(0-448.76)$ & $(0-405.29)$ \\
\hline 2. & 75.04 & $(0-223.80)$ & $(0-275.29)$ & $(0-250.21)$ \\
\hline & 71.04 & $0-187.91)$ & $(0-267.70)$ & $(0-225.12)$ \\
\hline 3. & 71.48 & $(0-180.32)$ & $(0-608.34)$ & $(0-411.03)$ \\
\hline 4. & \multicolumn{5}{|l|}{} \\
\hline
\end{tabular}

The Confidence Interval computation is:

$$
P\left[\hat{\bar{Y}} \pm 19.6 \sqrt{v(\hat{\bar{Y}}) / n^{2}}\right] \text {. }
$$

\section{CONCLUSION}

From above analysis it is clear that auxiliary variables play important role in the prediction of remaining processing time of ready queue. The length of confidence interval gets reduced if the auxiliary variables are selected and used in proper manner. Basically, any selected auxiliary variable may not provide the significant effect in time-length prediction. The variable which has higher correlation with the main variable (time) plays vital role and hence generates the lowest length confidence intervals in the setup of lottery scheduling. The suggested methodology is effective and efficient in predicting the queue processing remaining time if the sudden failure occurs in the job processing system.

\section{REFERENCES}

[1] Carl A. Waldspurger and William E. Weihl 1994 Lottery Scheduling a flexible proportional-share resource management. In Proceedings of the $1^{\text {st }}$ USENIX Symposium on Operating Systems Design and Implementation (OSDI), p1.

[2] Carl A. Waldspurger, Weihl, W. 1996 An Object-Oriented Framework for Modular Resource Management. In: Proceedings of the Fifth International Workshop on Object Orientation in Operating Systems, p 138.

[3] Cochran 2005 Sampling Technique, Wiley Eastern Publication, New Delhi.

[4] Shukla, D, Jain, Saurabh and Ojha, Shweta 2010 Effect of Data Model Approach for the Analysis of Multi-Level Queue Scheduling, International Journal of Advanced Networking and Applications, Vol. 2 Issue 1, p 419.

[5] Shukla,D. and Jain, Saurabh 2009 Analysis of Thread scheduling with multiple processors under a Markov chain model, Journal of Computer Science, Vol. 3 , Issue 5, p 78.

[6] Shukla,D., Jain, Saurabh and Singh, Umesh 2008 A Markov chain model for Deficit Round Robin Alternated (DRRA) scheduling algorithm. In Proceedings of the International Conference on Mathematics and Computer Science, ICMCS-08, p 52.

[7] David Petrou, Garth A. Gibson and John W. Milford 1999 Implementing Lottery Scheduling: Matching the specializations in Traditional Schedulers. In Proceedings of the USENIX Annual Technical Conference USA, p 66.

[8] Raz, D., B. Itzahak and H. Levy 2004 Classes, Priorities and Fairness in Queuing Systems, Research report, Rutgers University.

[9] Shukla D., Jain, Saurabh 2010 A Stochastic Model Approach for Reaching Probabilities of Message Flow in Space-Division Switches. International Journal of Computer Networks, Vol. 2, Issue 2, p 140.

[10] Shukla, D. and Jain, Saurabh 2007 A Markov chain model for multilevel queue scheduler in operating system. In Proceedings of International Conference on Mathematics and Computer Science, ICMCS-07, p. 522.

[11] Shukla, D. and Jain, Saurabh 2007 Deadlock state study in security based multilevel queue scheduling scheme in operating system. In Proceedings of National Conference on Network Security and Management, NCNSM-07, p 166.

[12] Shukla, D. Ojha, Shweta 2010 Analysis of multilevel queue with effect of data model approach. In Proceedings of National Conference on Research and Development Trends in ICT, 12-13 Feb., p 245.

[13] Shukla, D., Jain, Anjali 2010 Estimation of ready queue processing time under SL scheduling scheme in multiprocessor environment. International Journal of Computer Science and Security (IJCSS), Vol. 4(1), p 74.

[14] Shukla,D., Jain, Anjali and Choduary, Amita 2010 Estimation of ready queue processing time under Usual Group Lottery Scheduling(GLS) in Multiprocessor Environment. International Journal of Computer and Applications (IJCA), Vol. 8, No. 14, p 39.

[15] Shukla,D., Jain, Anjali and Choduary, Amita 2011 Estimation of ready queue processing time under Usual Lottery Scheduling(ULS) in Multiprocessor Environment. Journal of Applied Computer Science and Mathematics (JACSM), Vol. 11, No 11, p 39.

[16] Shukla,D. and Jain, Anjali 2012 Analysis of Ready Queue Processing Time under PPS-LS and SRS-LS scheme in Multiprocessing Environment. GESJ: Computer Science and Telecommunication, No.1 (33), pp. 54-61.

[17] Shukla,D. and Jain, Anjali 2012 Estimation of Ready Queue Processing Time Using Efficient Factor Type (E-F-T) Estimator in Multiprocessor environment, International Journal of Computer and Applications (IJCA), Vol. 48, No. 16 , pp. $20-27$. 
[18] Silberschatz, A. and Galvin, P. 1999 Operating System Concepts, Ed.5, John Wiley and Sons (Asia), Inc.

[19] Singh, Daroga and Choudhary, F.S. 1986 Theory and Analysis of Sample Survey and Designs, Wiley Eastern Limited, New Delhi.

[20] Stalling, W. 2004 Operating Systems, Ed.5, Pearson Education, Singapore, Indian Edition, New Delhi.

[21] Tanenbaum, A. 2000 Operating system, Ed. 8, Prentice Hall of India, New Delhi.

[22] Yiping Ding, William Flynn 2000 Interpreting Windows NT Processor Queue Length Measurements. In Proceedings of the 31st Computer Measurement Group Conference, Vol. 2, p 759 .
[23] Yiping Ding 2000 On the Performance Impact of Fair Share Scheduling. In Proceedings of the Computer Measurement Group Conference, p 71.

\section{Appendix}

The correlation coefficient

$$
\rho=\frac{E\left(y_{i}-\bar{Y}\right)\left(x_{i}-\bar{X}\right)}{\sqrt{E\left(y_{i}-\bar{Y}\right)^{2} E\left(x_{i}-\bar{X}\right)^{2}}}=\frac{\sum^{N}\left(y_{i}-\bar{Y}\right)\left(x_{i}-\bar{X}\right)}{(N-1) S_{y} S_{x}}
$$

